MATEC Web of Conferences 35, 06004 (2015)

DOI: $10.1051 /$ matec conf/20153506004

(C) Owned by the authors, published by EDP Sciences, 2015

\title{
Research on the System of Pulse Synthesis Pre-pump Rayleigh BOTDA technology
}

\author{
Qiufeng Shang ${ }^{1, a}$, Wengang Ren ${ }^{1}$, Xun Mao ${ }^{1}$ and Lixin Zhang ${ }^{1}$ \\ ${ }^{1}$ North China Electric Power University, Baoding, China
}

\begin{abstract}
The conventional Rayleigh scattering Brillouin Optical Time Domain Analysis (Rayleigh BOTDA) system adopted the leakage light ahead of the pump pulse to generate Rayleigh back-scattering light. Stimulated Brillouin scattering (SBS) interacts between an optical pulse and a Rayleigh back-scattering light, then the temperature and strain information of the optical fiber are perceived by measuring the Rayleigh scattering light. However, the width of pulse is inversely proportional to the accuracy of spectrum detection, which means the narrower the pulse, the wider the Brillouin spectrum. But the spatial resolution is strictly related to the duration of the optical pulse, when the pulse width is less than the phonon lifetime, the Brillouin spectrum width is much larger than the Brillouin natural gain spectrum width. The spectrum detection accuracy of the system can be effectively improved by adding a pre-pump ahead of the pump pulse to stimulate the acoustic wave field in the Rayleigh BOTDA system. In this paper, we present a new method of synthesizing step pulse. Through the OpiSystem software simulation and Matlab software simulation, the influence of the pre-pump width and power on the performance of the system is analyzed and the feasibility of this method is confirmed by preliminary experiment.
\end{abstract}

\section{Introduction}

Distributed optical fiber sensing technology has been developed in the last two decades. They permit to detect the change of strain and temperature and other physical quantities in different positions up to tens of kilometres using optical fiber sensing and transmitting signal so as to realize the real distributed measurement [1-4]. Compared with the traditional temperature measurement method, the distributed optical fiber temperature sensing system can simultaneously measure tens of thousands of points and accurately position each temperature measuring point.

In the traditional distributed optical fiber sensing configuration based on BOTDA, if the frequency difference between the pump light and counterpropagating continuous probe light which access to both ends of the fiber is equal to the Brillouin frequency shift, the probe signal is amplified at that point due to stimulated Brillouin scattering between the pump and probe light [5]. Therefore, it is possible to obtain the distributed temperature and strain information by measuring frequency shift of the probe light. The distributed optical fiber sensing technology based on Rayleigh scattering can obtain the distribution information of the measured physical quantities by measuring the Rayleigh back-scattering light. This configuration requires only one-end access to the sensing fiber but its sensing signal is weaker than the BOTDA system.
2011, Qingsong Cui et al employed the Rayleigh scattering light generated by the leakage light as probe light, producing stimulated Brillouin scattering effect between the pump and probe light, combining the Rayleigh scattering theory with BOTDA technique, obtaining the single end signal injected and high intensity sensing signal at the same time [6]. However, the spatial resolution is limited because of the width of pulse and the detection accuracy of the spectrum is restricted by each other. 2004, K. Kishida et al constructed the pulse prepump BOTDA with a pre-pump light ahead of the pulse light to excite the acoustic field so as to improve the accuracy of spectrum detection in the case of ensuring the spatial resolution [7].

In this paper, the pulse pre-pump technology is applied to the Rayleigh BOTDA system which has the advantages of single ended signal injection and detection, taking into account both the accuracy of spectrum detection and the spatial resolution. A new method for the synthesis of the pre-pump step pulse is proposed, the corresponding sensing system is constructed and the simulation analysis and preliminary experimental verification are carried out.

\section{System Configurations}

The overall block diagram of the system is depicted in Figure 1. Light from a laser with wavelength of $1.55 \mathrm{um}$ and line width of $1.6 \mathrm{MHz}$ is first spilt by a $3 \mathrm{~dB}(50: 50)$ optical coupler. In the upper branch, bias voltage

\footnotetext{
${ }^{\mathrm{a}}$ Corresponding author: lindashqf@126.com
} 


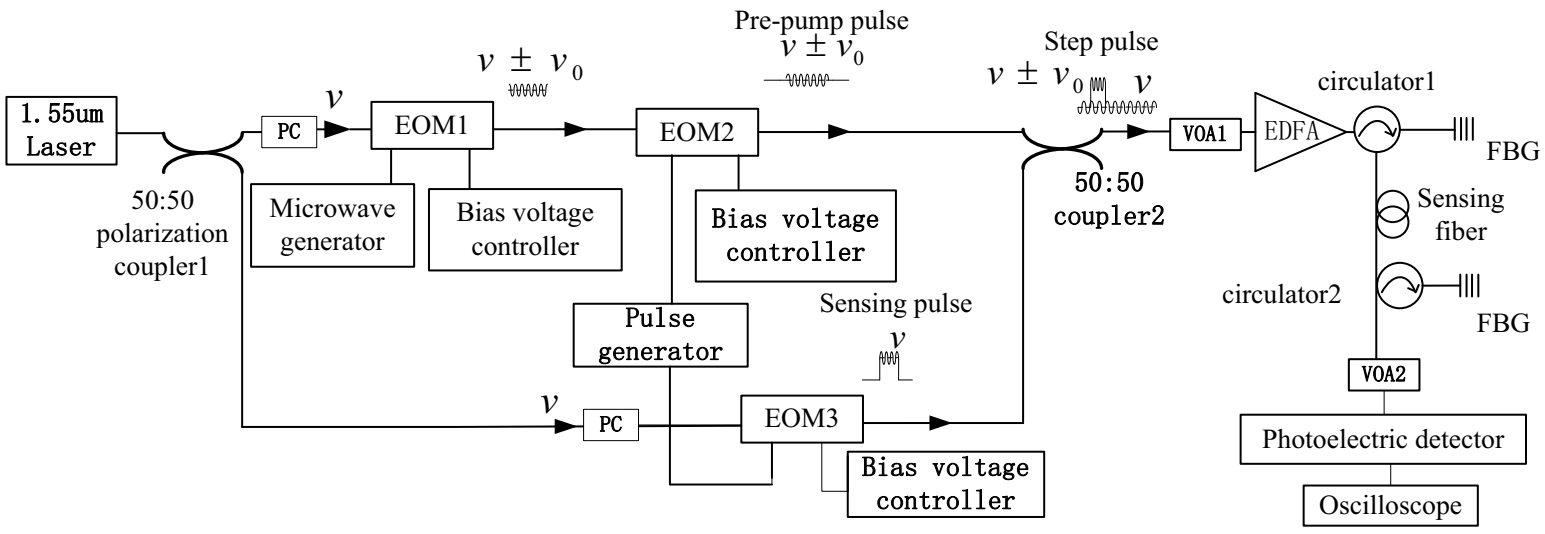

Figure 1. Experimental setup for pulse synthesis pre-pump Rayleigh BOTDA measurements

controller is connected to the bias port of Electro Optic Modulator (EOM1) and the microwave generator is connected to the RF port. The half wave voltage should be adjusted by the bias voltage controller to make the EOM1 work near the positive linear point, so that the light has 0 order baseband and 1 order sidebands. Then the light signal passed by EOM2. The pulse generator is connected to the bias port of EOM2 and the bias voltage controller is connected to the RF port. The bias controller controls EOM2 to work at the valley point, generating two pulse signals containing the sidebands by controlling the amplitude and width of the pulse. In the lower branch, the sensing pulse of the step pulse is generated through another Electro Optic Modulator (EOM3). The step pulse light is formed through coupler2 to converge the two branches, amplified by Erbium Doped Fiber Amplifier (EDFA), and then enter into the sensing fiber through the circulator.

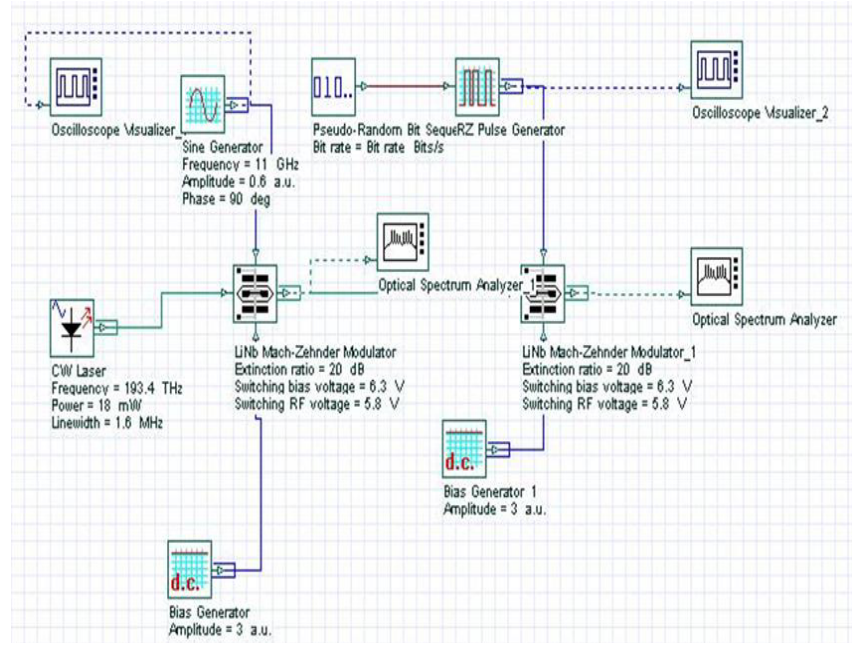

Figure 2. Optisystem simulating circuit diagram

The circuit diagram in the Optisystem system is showed in Figure 2. The output spectrum of EOM2 and EOM1 is observed by adjusting the parameters of EOM1 and EOM2 to optimize the working point of the step pulse as shown in Figure 3.

From Figure 3, the two EOM cascade can realize the synthesis of the 0 order baseband and 1 order sidebands of the light wave through the Optisystem software simulation.

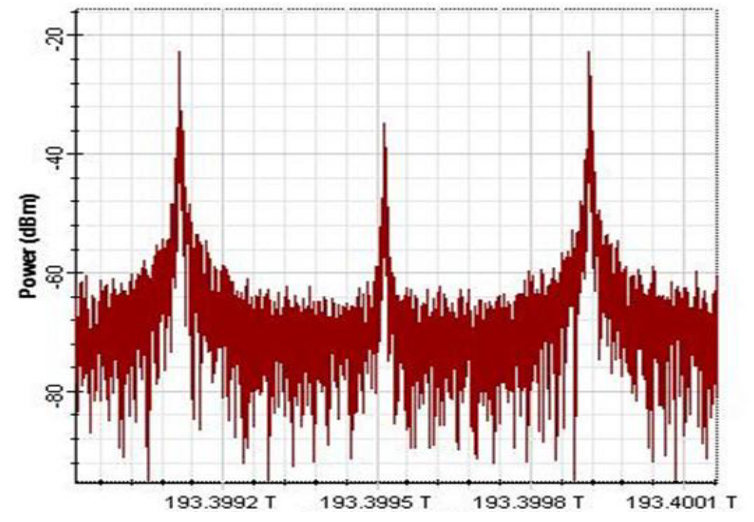

Figure 3. EOM output spectrum

\section{Theoretical Analysis}

In the step pulse modulated BOTDA system, Rayleigh scattering signal generated by the pre-pump pulse is used as probe light and the sensing pulse is used as pump light. Our model of the stimulated Brillouin scattering process uses the two cou-pled wave equations describing the two counter propagating lights [8]:

$$
\begin{aligned}
& \frac{d P}{d z}= \pm \frac{g}{A_{e f f}} P_{r} P-\alpha P \\
& \frac{d P_{r}}{d z}= \pm \frac{g}{A_{e f f}} P_{r} P+\alpha P_{r}
\end{aligned}
$$

In Equations (1-3), “土”denote two cases of Brillouin loss and Brillouin gain. $P_{r}(z)$ and $P(z)$ are respectively the Rayleigh scattering light and the sensing pulse light power at the fiber scattered point $\mathrm{z}, \mathrm{g}$ is the Brillouin gain coefficient, $A_{\text {eff }}$ is optical fiber effective area and $\alpha$ is the fiber loss coefficient.

The process of stimulated Brillouin gain between the Rayleigh light generated by the pre-pump pulse and sensing pulse is analyzed by using the transient analysis 
method of stimulated Brillouin steady state equation. The boundary conditions are the initial value of the pre-pump pulse is $\operatorname{Pp}(\mathrm{z}=0)=\mathrm{Pp}$ (power of the first order side band) and the initial value of the sensing pulse is $\mathrm{P}(\mathrm{z}=0)=\mathrm{P}$. Pre-pump pulse light is injected at the beginning of the optical fiber $\mathrm{z}=0$ and transmitted along the $+\mathrm{z}$ direction. The Rayleigh scattering optical power at position $\mathrm{z}$ is:

$$
P_{r}(z)=\frac{\alpha_{S} S}{2 \alpha} P_{p}[\exp (-\alpha z)-\exp (-\alpha(2 l-z))]
$$

Where $\alpha s$ is the Rayleigh scattering coefficient of $3.2 \times 10^{-2} / \mathrm{km}$ generally, $\mathrm{S}$ is the Rayleigh back-scattering light trapping coefficient of $1.5 \times 10^{-3}$ generally, 1 is the pre-pump pulse length. Substituting Equation (3) into (2) we get the power of sensing pulse light at position $\mathrm{z}$ as follows:

$$
P(z, v)=P \exp (-\alpha z) G(z, v)
$$

Where $G(z, v)$ is the sensing pulse light loss caused by SBS.

$$
\begin{aligned}
& G(z, v)=\exp \left[\int_{0}^{z}-\frac{g\left(z^{\prime}, v\right) P_{r}\left(z^{\prime}\right)}{A_{e f f}} d z^{\prime}\right] \\
& =\exp \left\{\frac{g(z, v) \alpha_{s} P_{p}}{A_{\text {eff }} \cdot 2 \alpha}\left[-\frac{\exp (-\alpha z)-1}{\alpha}+\frac{\exp (\alpha z-2 \alpha l)-1}{\alpha}\right]\right\}
\end{aligned}
$$

Then the Brillouin gain is calculated by integrating Equation (2) in the interval $\Delta \mathrm{z}=\mathrm{v}_{\mathrm{D}} / 2$. The result is:

$$
\begin{aligned}
& G^{\prime}(z, v)=\frac{P_{r}(z)}{P_{r}(z+\Delta z)} \\
& =\exp \left[\frac{g(z, v) P(z, v)}{A_{\text {eff }}} \Delta z\right] \exp (-\alpha \Delta z)
\end{aligned}
$$

When the pulse width is far less than the phonon lifetime, the transient Brillouin gain spectrum of the Rayleigh scattering light generated by the pre-pump pulse is required. For short pulse, the effect of the pulse spectrum characteristic on the Brillouin gain spectrum is considered. Convoluting the Brillouin gain in steady state $g(z, v)=\gamma g_{0}\left(\Delta v_{B} / 2\right)^{2} /\left[\left(v-v_{B}\right)^{2}+\left(\Delta v_{B} / 2\right)^{2}\right]$ and the pulse power spectrum, then the transient Brillouin gain is obtained as follows:

$$
\begin{aligned}
& g_{t}(z, v)=g(z, v) * f(v) \\
& =\gamma g_{0} \cdot \frac{1}{A^{2}+1} \cdot\{1+ \\
& \left.\frac{\left(A^{2}-1\right)-\exp (-B)\left[\left(A^{2}-1\right) \cos (B A)+2 A \sin (B A)\right]}{B\left(A^{2}+1\right)}\right\} \\
& =\gamma g_{0} \cdot F(A, B)
\end{aligned}
$$

Where $A=\left(v-v_{B}\right) /\left(\Delta v_{B} / 2\right), v$ is the optical frequency, $v_{B}$ is the Brillouin frequency shift, $\Delta v_{B}$ is the Brillouin natural gain spectrum width, $B=\pi D \Delta v_{B}, \mathrm{~g} 0$ is the Brillouin peak gain and $\gamma$ is the polarization factor dependent on the polarization state of the sensing pulse and the Rayleigh scattering light.
Replacing $g(z, v)$ with $g_{t}(z, v)$ in Equation (5) and (6), then the Brillouin gain of the SBS effect on the Rayleigh scattering light generated by the pre-pump pulse and the sensing pulse is:

$$
\begin{aligned}
& G_{t}^{\prime}(z, v) \\
& =\exp \left[\frac{g_{t}(z, v) P_{t}(z, v)}{A_{\text {eff }}} \Delta z\right] \exp (-\alpha \Delta z)
\end{aligned}
$$

Where $P_{t}(z, v)$ is the power of the sensing pulse in the transient analysis.

Neglecting the influence of the pre-pump pulse before the circulator, the power of Rayleigh scattering light along the fiber distribution generated by the sensing pulse light detected at the start end of the optical fiber is:

$$
P_{r}(z)=\frac{\alpha_{S} S}{2 \alpha} P_{p}[\exp (-\alpha z)-\exp (-\alpha(2 l-z))]
$$

When the power of step pulse light is $P_{t}(z, v)$ and the base band power of the pre-pump pulse is $P(z, v)$, the Brillouin scattering spectrum of the step light can be obtained as follows:

$$
P=P_{r}(z) \times G^{\prime}(z, v) \times G_{t}^{\prime}(z, v)
$$

Equation (10) is the Brillouin gain of Rayleigh scattering light. The Lorenz curve of the probe and the relationship between the temperature strain of the optical fiber and the frequency shift can be obtained by changing the frequency $v$.

\section{Simulation and Experimental Analysis}

Brillouin scattering gain intensity of the Rayleigh scattering light generated by the step pulse is affected by the width and power of the step pulse. In order to obtain the optimal relationship between the signal intensity and the pulse width and the power of the step pulse, the model is analyzed and simulated as follows.

Simulations have been carried out by considering a standard single mode optical fiber with the length of $10 \mathrm{~km}$, the Brillouin frequency shift of $10.85 \mathrm{GHz}$ and the Brillouin natural gain spectrum width of $35 \mathrm{MHz}$. According to Equation (5) and (10), the relationship between different width and power of the pre-pump pulse and the width of the Rayleigh scattering spectrum is obtained by changing width and the peak power of the pre-pump pulse, which is shown in Figure 4. 


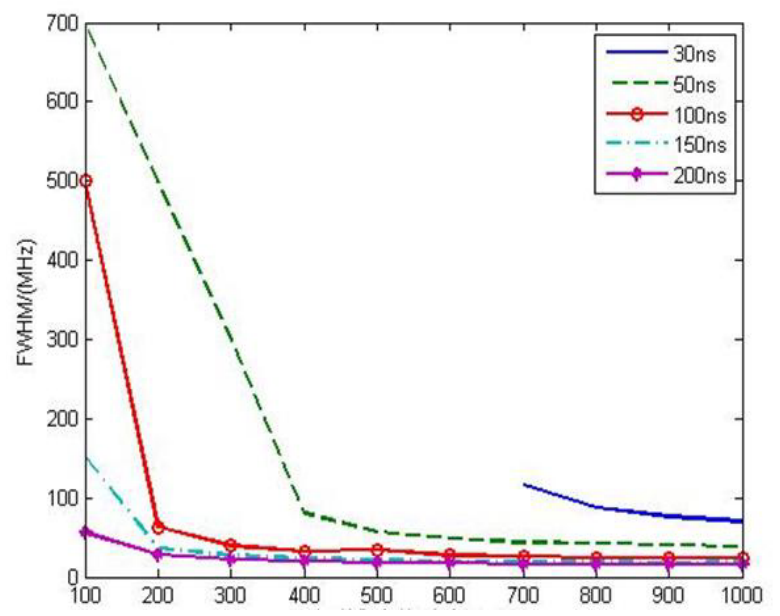

Figure 4. (a) Relationship between Brillouin spectrum width and power of pre-pump (horizontal axis $/ \mathrm{mW}$ ).

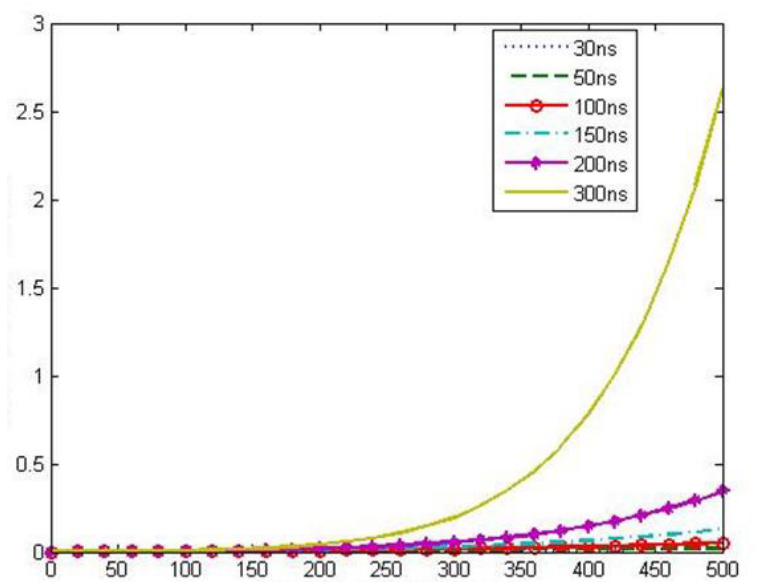

Figure 4. (b) Relationship between peak power of Brillouin spectrum (vertical axis $/ 0.1 \mathrm{~mW}$ ) and peak power of pre-pump (horizontal axis $/ \mathrm{mW}$ )

From Figure 4, we can see that the FWHM is $34 \mathrm{MHz}$ in case of the base band of $500 \mathrm{~mW}$ and the pre-pump pulse width of $100 \mathrm{~ns}$, which meets the accuracy requirements of frequency shift measurement according to Equation (10). Considering that the thermal noise, spontaneous noise and other noises exist in the optical fiber, we choose the sensing pulse power of $50 \mathrm{~mW}$ in order to meet the signal to noise ratio that experiment required. This system is expected to achieve the spatial resolution of $50 \mathrm{~cm}$, so the width of the injected pulse is determined to be 5 ns.

Then the experimental study is carried out. We test out the waveform of the synthetic pulse firstly. Before injected into the optical fiber, the pulse waveform should be observed. The synthetic pre-pump waveform is observed through the oscilloscope as Figure 5.

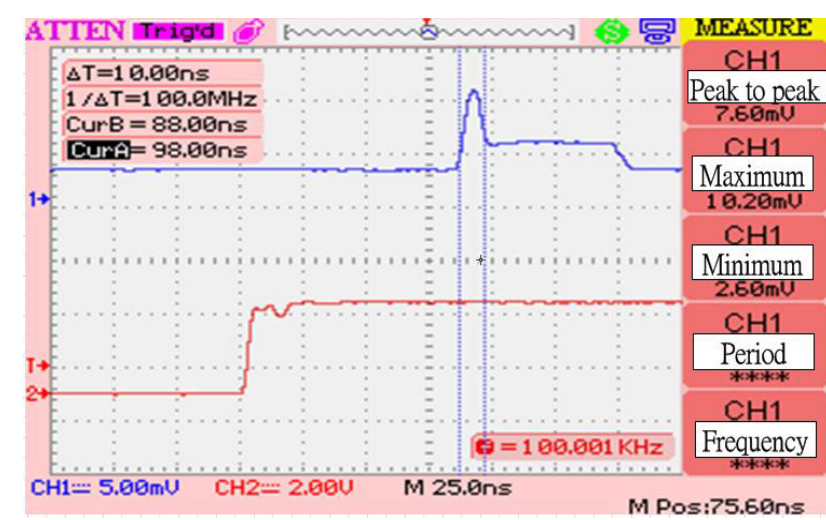

Figure 5. The synthetic pre-pump waveform

Then observation of Rayleigh scattering signal according to the proposed system, setting the center frequency of $10.868 \mathrm{GHz}$. After the optical is scattered back to the photoelectric detector, the power of Rayleigh scattering signal in one period can be observed by the oscilloscope as Figure 6.

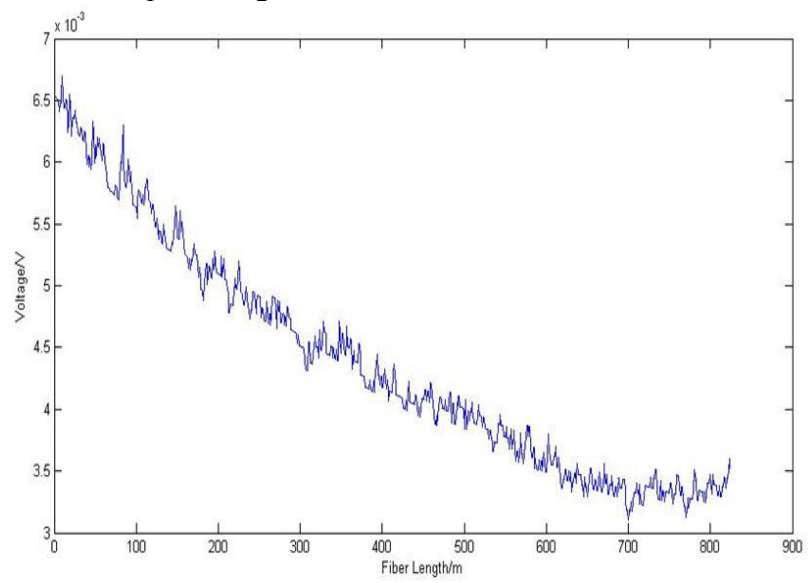

Figure 6. Rayleigh scattering waveform

From Figure 6, we can see that this system can realize the observation of the optical fiber signal.

\section{Summary}

This paper presents a new method of synthesizing step pulse, which means adding a pre-pump ahead of the pump pulse to stimulate the acoustic wave field and generate the desired Rayleigh scattering light in the BOTDA system, effectively solving the mutual restriction problem between the spatial resolution and the pulse width. The system can realize the distributed sensing of optical fiber with a single end incident due to using the Rayleigh scattering light as probe. As a result, the distributed sensing system can be realized by using a single incident light. Through theoretical simulation, we find that when the pulse width is $5 \mathrm{~ns}$, the power is $50 \mathrm{mw}$, the pre-pump width is $100 \mathrm{~ns}$ and the power is $500 \mathrm{~mW}$, the best accuracy of spectrum measurement can be obtained. 


\section{Acknowledgments}

This study is supported by Nature Science Foundation of Hebei Province (F2014502098) and National Nature Science Foundation of China (61377088, 51407074).

\section{References}

1. A. Minardo, R. Bernini, Zeni, A Simple technique for Reducing Pump Depletion in Long Range Distributed Brillouin Fiber Sensors. Sensors Journal 9, 7(2009)

2. E. Geinitz, S. Jetschke, The influence of pulse amplification on distributed fibre-optic Brillouin sensing and a method to compensate for systematic errors, Meas. Sci. Technol 10 (1999)

3. R. Bernini, A. Minardo, L. Zeni A Reconstruction Technique for Stimulated Brillouin scattering fiberoptic sensors for simultaneous measurement of temperature and strain, IEEE 2 (2002)

4. Zornoza A, Minardo et al, Pulsing the Probe Wave to Reduce Nonlocal Effects in Brillouin Optical TimeDomian Analysis Sensor, Sensors Journal 11 (2011)

5. Qingsong Cui et al, Distributed fiber sensor based on modulated pulse base reflection and Brillouin gain spectrum analysis, Optical Society 5 (2009)

6. Qingsong Cui, S. Pamukcu et al, Distributed temperature sensing system based on Rayleigh scattering BOTDA, IEEE 11 (2011)

7. K. Kishida et al, Pulsed pre-pump method to achieve cm-order spatial resolution in Brillouin distributed measing technique, IEICE 10 (2004)

8. Luc Thévenaz et al, Effect of pulse depletion in a Brillouin optical time-domain analysis system, Optical Society 6 (2013) 